\title{
Design of a novel phase-decoupling permanent magnet brushless ac motor
}

\author{
Wei Cui \\ Department of Electrical \& Electronic Engineering, The University of Hong Kong, Hong Kong, China and \\ Department of Automation, Shanghai University, Shanghai, China \\ K. T. Chau \\ Department of Electrical \& Electronic Engineering, The University of Hong Kong, Hong Kong, China \\ J. Z. Jiang \\ Department of Automation, Shanghai University, Shanghai, China \\ Ying Fan \\ Department of Electrical \& Electronic Engineering, The University of Hong Kong, Hong Kong, China
}

(Presented on 11 November 2004; published online 17 May 2005)

\begin{abstract}
This paper presents a phase-decoupling permanent magnet brushless ac motor which can offer better controllability, faster response, and smoother torque than its counterparts. The key is due to its different motor configuration and simple scalar control. The motor configuration is so unique that it inherently offers the features of phase decoupling, flux focusing, and flux shaping, hence achieving independent phase control, fast response, and smooth torque. The scalar control is fundamentally different from the complicated vector control. It can achieve direct torque control through independent control of the phase currents. The proposed motor is prototyped and experimentally verified. (C) 2005 American Institute of Physics. [DOI: 10.1063/1.1860833]
\end{abstract}

\section{INTRODUCTION}

With the invention of neodymium-iron-boron (Nd$\mathrm{Fe}-\mathrm{B})$ magnets, the development of permanent magnet (PM) brushless motors is accelerating. ${ }^{1}$ Those viable types of PM brushless motors are the PM brushless ac (PMBAC) motor, ${ }^{2}$ PM brushless dc motor, ${ }^{3}$ doubly salient PM motor, ${ }^{4}$ and PM hybrid motor. ${ }^{5}$ The PMBAC motor inherently offers the advantages of high efficiency and high power density. However, its controllability, transient response, and torque smoothness need to be improved to satisfy the requirements for high-performance applications.

The purpose of this paper is to design and implement a PMBAC motor which not only retains its inherent advantages, but also offers better controllability, faster response, and smoother torque than its counterparts.

\section{DESIGN AND ANALYSIS}

As shown in Fig. 1, the proposed motor offers three unique features, namely, the phase-decoupling stator windings, the flux-focusing PM rotor, and the flux-shaping air gap.

First, the coil span of stator windings is purposely selected to be one slot pitch (for example, a coil of phase $A$ spans between the slot 1 and slot 2) so that the flux paths of different phases are independent. Hence, the mutual couplings or inductances between phases are practically eliminated, so-called the phase decoupling. By applying finite element analysis, both self- and mutual inductances can be calculated. Figure 2 shows the inductances at the rated current, confirming that the mutual inductance $(0.043 \mathrm{mH})$ is negligible compared with the self-inductance $(0.94 \mathrm{mH})$. With the nature of phase decoupling, there is no need to perform the complicated coordinate transformation for vec- tor control. Thus, both the controllability and transient response can be significantly improved with the use of simple scalar control.

Second, all magnets are buried inside the rotor in such a way that the flux density of each pole is contributed by two radially located (main) magnets and one circumferentially located (auxiliary) magnet, so-called the flux focusing. Figure 3 shows the air-gap flux density distributions with and without using the auxiliary magnet. It confirms that this flux focusing can remarkably increase the air-gap flux density, hence the transient response can be significantly improved.

Third, instead of using stator or rotor skewing, ${ }^{6}$ the proposed motor adopts the eccentric air gap to improve the torque smoothness. It is based on the variation of air-gap thickness $\delta$, namely, the minimum at the pole central whereas the maximum at its two ends, to shape the air-gap flux density wave form, so-called the flux shaping. Figure 3

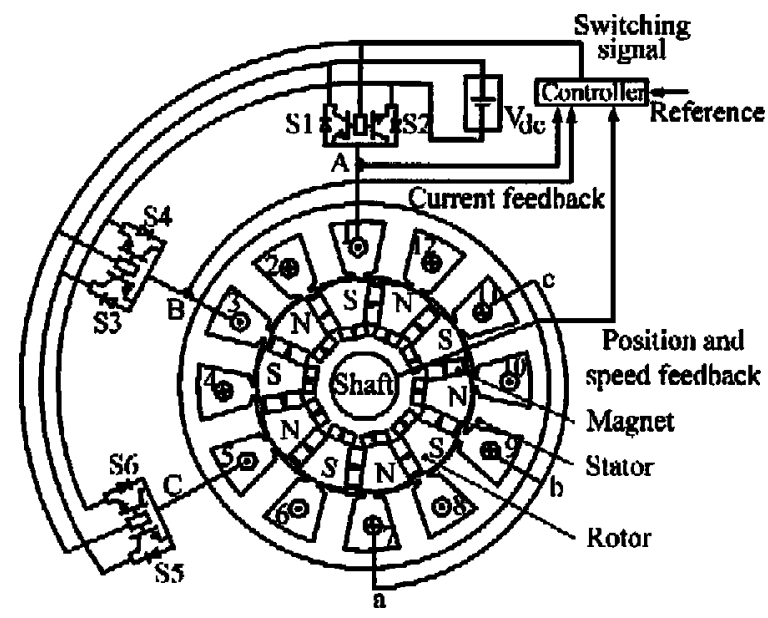

FIG. 1. Proposed motor configuration. 


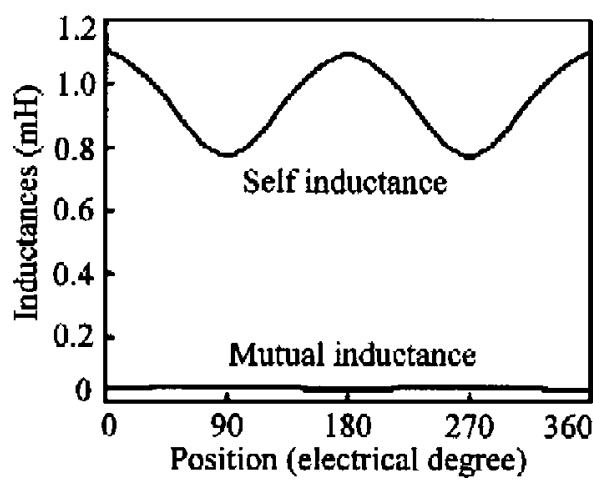

FIG. 2. Calculated inductances at rated current.

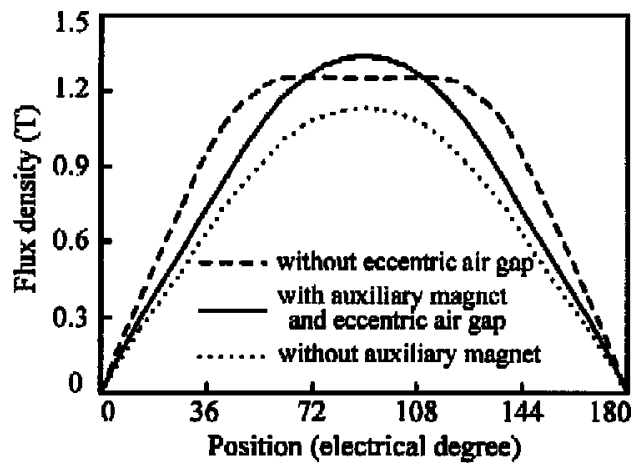

FIG. 3. Air-gap flux density distributions.

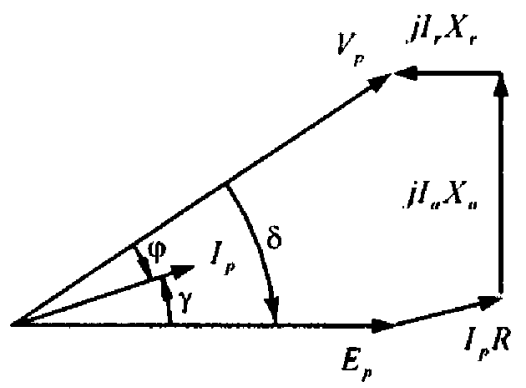

FIG. 4. Scalar control operation.

TABLE I. Motor specifications.

\begin{tabular}{lc}
\hline \hline \multicolumn{1}{c}{ Parameters } & Data \\
\hline Rated dc-link voltage & $24 \mathrm{~V}$ \\
Rated phase current & $13 \mathrm{~A}$ \\
Rated torque & $4.5 \mathrm{~N} \mathrm{~m}$ \\
Rated speed & $600 \mathrm{rpm}$ \\
No. of phases & 3 \\
No. of slots & 12 \\
No. of coils & 6 \\
No. of poles & 10 \\
\hline \hline
\end{tabular}
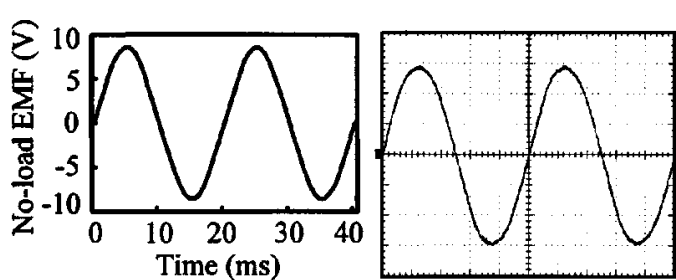

FIG. 5. Comparison of simulated and measured no-load emf wave forms at rated speed $(3 \mathrm{~V} / \mathrm{div}, 4 \mathrm{~ms} / \mathrm{div})$.

also shows the air-gap flux density distributions with and without adopting eccentric air gap. It confirms that when $\delta_{\max }=1.22 \mathrm{~mm}$ and $\delta_{\min }=0.35 \mathrm{~mm}$, it can yield the most sinusoidal air-gap flux density and hence the smoothest torque.

\section{CONTROL AND OPERATION}

Because of the nature of phase decoupling, the proposed motor offers a definite advantage that the current of each phase winding can be independently controlled. Hence, a simple scalar control of the phase current amplitude and its angle with respect to the back emf can be devised for direct torque control. Complex coordinate transformation and computation are no longer necessary. Figure 4 illustrates how to control the phase current and hence the torque, namely, $\gamma$ $=0$ for constant-torque operation whereas $\gamma \neq 0$ for constantpower operation. The corresponding torque expression can be analytically derived as

$$
T_{e}=\frac{3 p}{\omega}\left(E_{p} I_{p} \cos \gamma+X_{2} I_{p}^{2} \sin 2 \gamma\right),
$$

where $p$ is the number of pole-pairs, $\omega$ is the supply angular frequency, $E_{p}$ is the phase emf, $I_{p}$ is the phase current, $\gamma$ is the phase shift between $E_{p}$ and $I_{p}$, and $X_{2}$ is the second-order harmonic amplitude of the self-inductance.

\section{SIMULATION AND EXPERIMENTATION}

The proposed motor is prototyped for experimentation. Table I lists its specifications. This motor is purposely designed for application to a low-voltage battery-powered electric motorcycle.

Figure 5 shows both the simulated and measured no-load emf wave forms at $600 \mathrm{rpm}$. The corresponding agreement is very good with nearly the same amplitude of $8.6 \mathrm{~V}$. Figures 6 and 7 epict both the simulated and measured phase current wave forms for constant-torque operation at $600 \mathrm{rpm}$ with $\gamma=0^{\circ}$ and constant-power operation at $1200 \mathrm{rpm}$ with $\gamma$ $=40^{\circ}$, respectively. As expected, the agreements are also
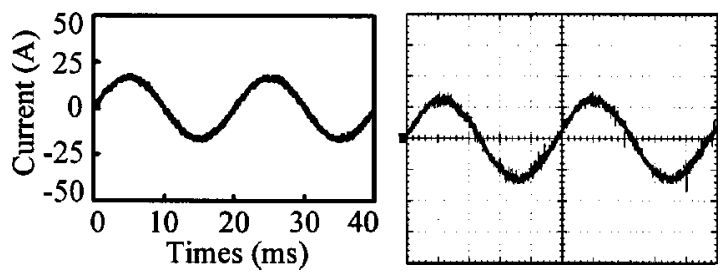

FIG. 6. Comparison of simulated and measured current wave forms at constant-torque operation (14 A/div, $4 \mathrm{~ms} / \mathrm{div})$. 

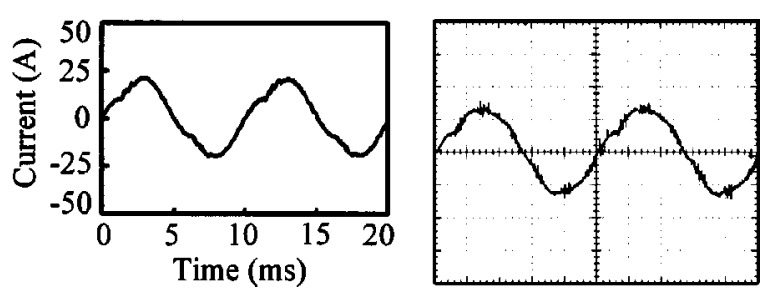

FIG. 7. Comparison of simulated and measured current wave forms at constant-power operation (14 A/div, $2 \mathrm{~ms} / \mathrm{div})$.

very good, namely, the amplitude of 18.9 A (simulated) versus 18.2 A (measured) when $\gamma=0^{\circ}$; and 19.9 A (simulated) versus 18.2 A (measured) when $\gamma=40^{\circ}$. Since both the emf and current wave forms are very sinusoidal, the resulting torques are very smooth for both constant-torque and constant-power operations.

Moreover, Fig. 8 shows the simulated and measured start-up transient responses at no-load. It can be found that the agreement is very good with nearly the same steady-state speed of $600 \mathrm{rpm}$, steady-state current of $1.7 \mathrm{~A}$, and rise-up time of $40 \mathrm{~ms}$. Such rise-up time verifies its merit of fast response. Nevertheless, there are some minor discrepancies in the overshoot current of $10 \mathrm{~A}$ (simulated) versus $9.5 \mathrm{~A}$ (measured), and the undershoot current of $-6.1 \mathrm{~A}$ (simulated) versus $-7.8 \mathrm{~A}$ (measured). These discrepancies are mainly due to the inevitable difference between theoretical and actual motor parameters.

Finally, the simulated and measured torque-speed capabilities are shown in Fig. 9. The agreement confirms that the
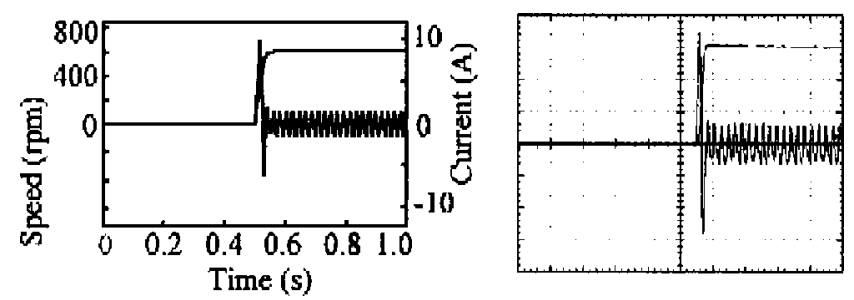

FIG. 8. Comparison of simulated and measured speed and current responses at no-load start-up (200 rpm/div, $2.8 \mathrm{~A} / \mathrm{div}, 100 \mathrm{~ms} / \mathrm{div})$.

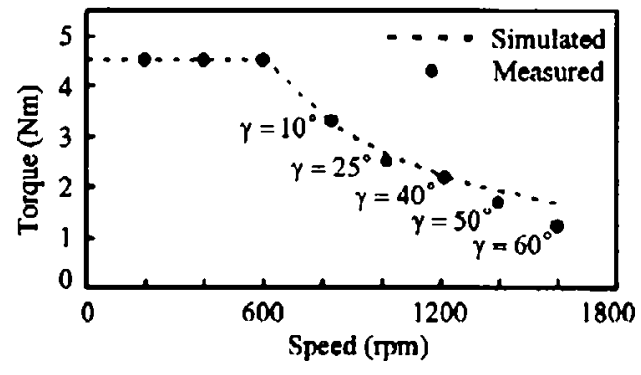

FIG. 9. Comparison of simulated and measured torque-speed capabilities.

proposed motor can offer constant-torque operation at the rated torque of $4.5 \mathrm{Nm}$ and constant-power operation up to $1600 \mathrm{rpm}$. There are some discrepancies at speeds beyond $1200 \mathrm{rpm}$, which are mainly due to the increasing significance of nonlinear windage and frictional torques at high speeds.

\section{CONCLUSION}

In this paper, a PMBAC motor has been designed and implemented for a low-voltage battery-powered electric motorcycle. The proposed motor not only retains the inherent advantages of PMBAC motors, but also offers better controllability, faster response, and smoother torque than its counterparts. Theoretical discussion, computer simulation, and experimental verification are provided for illustration.

\section{ACKNOWLEDGMENT}

This work was funded by Research Grants Council of Hong Kong, China (Project No. HKU 7035/01E).

${ }^{1}$ T. M. Jahns, Proc. IEEE 82, 1241 (1994).

${ }^{2}$ C. C. Chan and K. T. Chau, IEEE Trans. Veh. Technol. 45, 180 (1996).

${ }^{3}$ J. Gan, K. T. Chau, C. C. Chan, and J. Z. Jiang, IEEE Trans. Magn. 36, 3810 (2000).

${ }^{4}$ M. Cheng, K. T. Chau, and C. C. Chan, IEEE Trans. Magn. 37, 3012 (2001).

${ }^{5}$ K. T. Chau, J. Z. Jiang, and Y. Wang, IEEE Trans. Magn. 39, 3001 (2003). ${ }^{6}$ Z. Q. Zhu, S. Ruangsinchaiwanich, N. Schofield, and D. Howe, IEEE Trans. Magn. 39, 3238 (2003). 